\section{Standardized data collection to build prediction models in oncology: a prototype for rectal cancer}

\author{
Elisa Meldolesi', Johan van Soest ${ }^{2}$, Andrea Damiani', Andre Dekker², Anna Rita \\ Alitto', Maura Campitelli', Nicola Dinapoli', Roberto Gatta', Maria Antonietta \\ ${\text { Gambacorta }{ }^{*}, 1}$, Vito Lanzotti', Philippe Lambin ${ }^{2}$ \& Vincenzo Valentini'
}

\begin{abstract}
The advances in diagnostic and treatment technology are responsible for a remarkable transformation in the internal medicine concept with the establishment of a new idea of personalized medicine. Inter- and intra-patient tumor heterogeneity and the clinical outcome and/or treatment's toxicity's complexity, justify the effort to develop predictive models from decision support systems. However, the number of evaluated variables coming from multiple disciplines: oncology, computer science, bioinformatics, statistics, genomics, imaging, among others could be very large thus making traditional statistical analysis difficult to exploit. Automated data-mining processes and machine learning approaches can be a solution to organize the massive amount of data, trying to unravel important interaction. The purpose of this paper is to describe the strategy to collect and analyze data properly for decision support and introduce the concept of an 'umbrella protocol' within the framework of 'rapid learning healthcare'.
\end{abstract}

First draft submitted: 14 July 2015; Accepted for publication: 26 October 2015; Published online: 17 December 2015

In the last two decades, the progressive and continuous effort to develop predictive models in oncology field, shows the urgency to be able to combine the tremendous advances in diagnostic and treatment technology with the advances in the individualized medicine [1-7]. The possibility, for treating physicians, to incorporate knowledge available in validated decision support systems (DSSs) beside the widely accepted and daily used clinical guidelines, can makes treatments more and more individualized for each patient, offering a valuable assistance in clinical decision-making in daily practice $[8]$.

Moreover, the current worldwide economic situation requires special care in new technology and pharmacology adoption asking for a deep knowledge of the benefits of new drugs or technologies for a reliable and cost-effective address of the resources [9].

The role and value of well-structured randomized clinical trials and subsequent systematic reviews or meta-analyses cannot be ignored in the definition of the clinical guidelines. Besides that, the continuing request to evaluate new drugs or treatment strategies, the possibility to enroll only selective subgroups of general population and the high heterogeneity (in terms of outcomes, methodology, patient's characteristics, data storing systems, among others) between different studies, justifies the progressive emergence of population-based observational studies as a complementary form of research, often named 'rapid learning healthcare' (RLHC), beside randomized clinical trials [10-12]. Observational studies are essential to ensure that the result of clinical trials translate

'Radiotherapy Department, Sacred Heart University, Rome, Italy

2Department of Radiation Oncology (MAASTRO), GROW-School for Oncology \& Developmental Biology, Maastricht University Medical Centre, Maastricht, The Netherlands

*Author for correspondence: Tel.: +39063015 5534; magambacorta@rm.unicatt.it
KEYWORDS

- Big Data • data standardization • decision support system • ontology - predictive models

- semantic web • umbrella protocol 
into tangible benefits in the general population, identifying whether practice has changed appropriately in a wider population with different characteristics [10]. One of the main criticism of RLHC approaches is the low-data quality. We hypotesize that the use of standardized data collection (SDC) will improve the quality of collected data.

\section{Multifactorial predictive models: a continuing challenge}

Nowadays, it is well known how the complexity of the clinical outcome and/or the treatment's toxicity is influenced by factors that can hardly be summarized by the few variables that the limited human cognitive capacity is able to discriminate and use in a daily clinical practice [13]. The key point of the different behavior of the same tumor in different patients is more and more likely due to the numerous interactions between multiple patients' specific factors. The need to acquire and comprehend patient's characteristics has created in the past new challenges. Clinical factors have been combined with multiple different variables coming from the remarkable expansion of several fields as the medical imaging technologies, pathology, biologic biomarkers, genomics, proteomics, among others. However, in many cases, the number of evaluated features could be very large resulting in statistical challenges in mono-centric analysis. In these situations, either (semi-)automated data-mining or machine learning processes can be a good solution for Big Data analyses, able to unravel important complex, nonlinear interactions among input and outcome variables; comprising the potential to predict undiscovered information for prospective clinical applications $[14,15]$. The role of radiation in this even more complex oncological domain has been widely examined in the last years $[16,17]$. On the other hand, the tremendous progress in technology (for instance, the development of new hightech devices that allow multiple advanced dose modulation and delivery techniques), introduce complexities when comparing treatment modalities; including the high heterogeneity of dose distribution in terms of both irradiated normal tissues and target volumes. Furthermore, considered to be a physical intervention, radiation therapy is actually more a biological intervention with effects at the cellular and molecular level explaining the growing efficacy seen in the combination with targeted biological agents.
Several interactive DSSs have been developed in the last years related to different oncologic diseases (Table 1) [18-21]. To the best of our knowledge none are certified and clinical grade (CE marked of US FDA approved)

Recently, the reporting quality of prediction model studies has been recently questioned through the 'Transparent Reporting of a multivariable prediction model for Individual Prognosis Or Diagnosis (TRIPOD)' initiative. In most of the analyzed studies, reporting was found to be poor, with insufficient information regarding the development and validation of the models from the description of patients' data to statistical modeling methods. Based on their analysis, the TRIPOD Statement, a set of recommendations to improve the transparency of studies reporting on develop or validation of prediction models, has been developed [29].

Nevertheless, these models are often used in clinical practice beside the existing consensus and/or guidelines: medical doctors and/or patients can use them into a 'shared decision-making' process where doctors and patients actively discuss and decide on therapeutic interventions, being able to choose the better treatment strategy for each single patient. Predictive models can be represented in a variety of ways. Graphical calculating devices as nomograms $[1,30]$ are one of the most common form of predictive device, beside the even more appealing interactive website. Furthermore, in this era of technological progress, is also very interesting the possibility to create specific applications for devices of new generation (e.g., cell phones, tablet, among others). Moreover DDS can be integrated into electronic medical records (EMR) in order to limit the double dataentry and assist the user with warnings, reminders by automatic check of the stored clinical pathway. However, this remains an active field of research in terms of alerts/information presented and actual use in the clinical workflow [31].

Notwithstanding this explosion of knowledge and the development of high selective treatment therapies, the early clinical data are still, in some cases, largely disappointing with large areas of controversies that still exist in the daily practical patient's care $[32,33]$, justifying the high interest in the development of multifactorial predictive models.

\section{Ontology \& data standardization}

The methodological process to develop a DSS based on validated predictive models has been 
deeply explained by Lambin et al. [11,34]. This new concept of research implies that all the information has to be collected without knowing beforehand what the final relevant features will be. A large heterogeneous database is the result of this multi-departmental and/or multiinstitutional data collection and implies a standardization of any considered variable in order to universally define data and procedures allowing automatic and consistent upload from any exploitable data source.

This SDC process will take advantage of an ontology that formally represents a system where each variable within a domain of interest can be classified in an uniform and unambiguous way. This system is able to define not only the characteristics of each single variable, but also their relationships. Hence, building a contract and/or consensus on the specification of terminologies and relationships used for a given domain is needed $[35,36]$. Additionally, specifying the provenance of data in, for example, geographical (e.g., between different institutions) and temporal (e.g., version of classificatios) aspects, add opportunities in terms of secondary use and comparability of clinical data.

Furthermore, this kind of data collection model should be open also to the necessity of extending the number of potential variables to collect over time to be able to comprehend all the clinical, treatment and technical advances. The possibility to have Big high-quality Data in digital format can also make it possible to create a RLHC system to address clinical problems thanks to the creation of an open source research protocol repository [37].

From the purely qualitative point of view of computer science, the formalization of any ontology can grow from a simple dictionary, where the meaning of the terms is described in natural language, toward a more and more formal expression resulting also from the sharing of the definitions between different institutions on a local, national or international level [35]. At the cost of increasing complexity and formalism that enriches the language with more and more complex constructs representing relationships between variables, different techniques can be used for representing richer knowledge contents (Figure 1) [38].

SDC will improve the quality of the collected data by defining which variables should be collected and how and when these variables should be measured. All the information will be collected and organized in any ontology starting
Table 1. Examples of interactive decision support systems currently in use in clinical practice.

Institution Ref.

European Organization for Research and Treatment of Cancer (EORTC)

Memorial Sloan Kettering Cancer Center (MSKCC)

Dana Farber Cancer Institute and Johns Hopkins Sidney Kimmel Comprehensive

Cancer Center

Massachusetts General Hospital (MGH)

Cancer Research UK

National Cancer Institute ( $\mathrm{NCl}$ )

Maastro Clinic

from a generic 'registry' layer with exclusively epidemiologic information, to a 'procedural' level, where treatment information and related toxicities are reported, up to a higher 'research' level where dimensional data, such as images, genomics, proteomics, among others, are collected [39]. Therefore, in the development of an ontology, the information can grow both in terms of variety and granularity, towards the idea of a large clinical database trying to capture and comprehend all information available from the patient.

\section{Umbrella protocol}

The strategy to collect data in a standard and consistent manner and to analyze them properly for decision support is called 'umbrella protocol' (Figure 2) [39]. The umbrella protocol is a new concept to facilitate RLHC programs and contains a set of materials and procedures related to a group of specific research projects [11]. All the information related to a specific domain (e.g., rectal cancer) is described in an ontology without knowing beforehand what the most relevant features for specific hypothesis will be. The main SDC strategies, the analysis procedures and the possible occurring ethical issues are described in an umbrella protocol. An umbrella protocol tries to be as complete as possible accompanying ontology for a specific domain (trying to capture everything under the 'umbrella' for this domain) and embracing current evidence and outcomes. An umbrella protocol related to the rectal cancer's SDC has been recently drafted in the radiation oncology department of the Policlinico A Gemelli in Rome. The main steps are described in the following paragraphs.

\section{A rectal cancer's SDC prototype}

During the last few years, research activity, related mainly to rectal cancer, has been very active inside our Radiation Oncology Department: Gemelli 


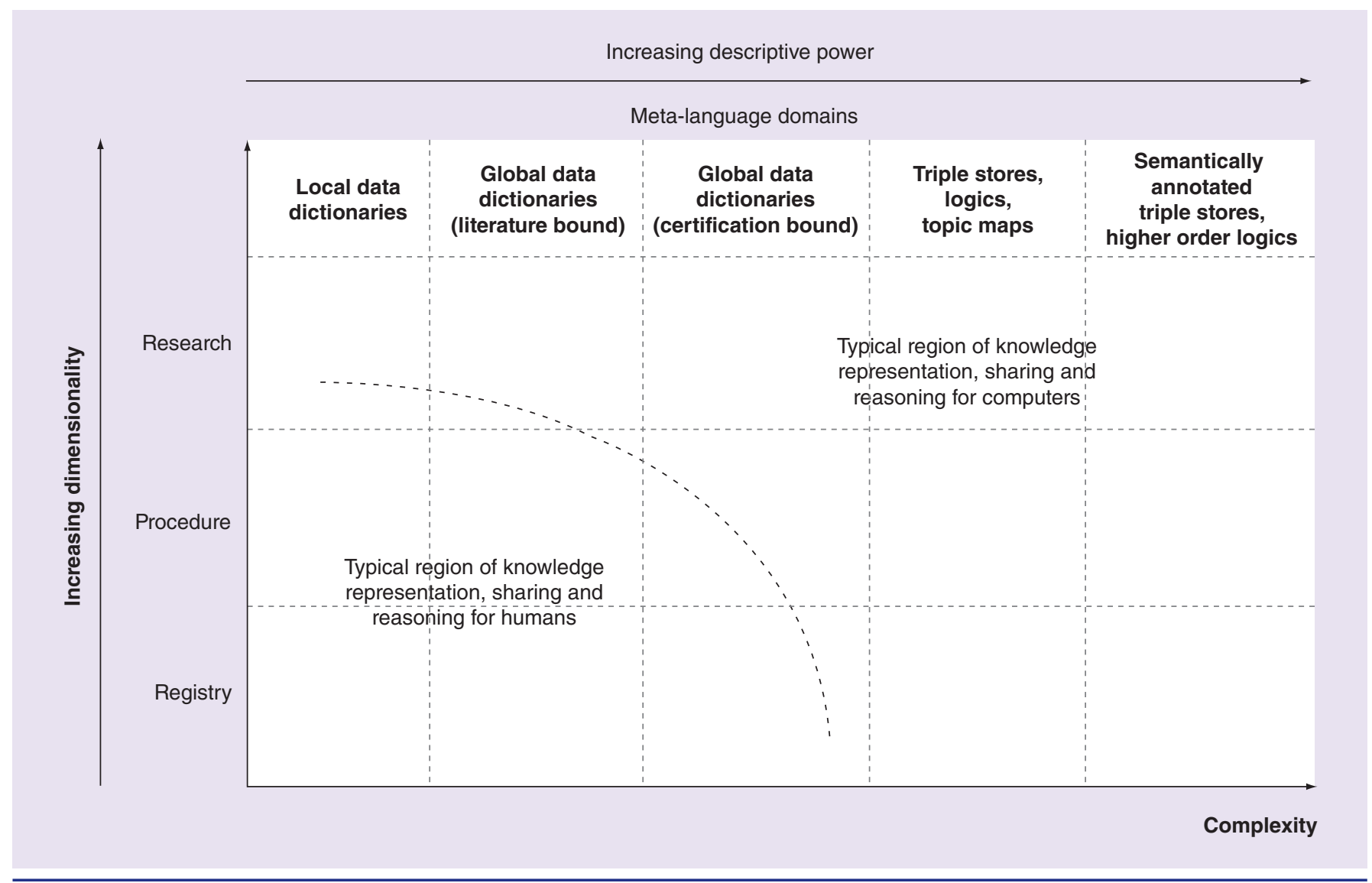

Figure 1. Possible evolution in knowledge representation, seen from the perspective of computer science, under a qualitative point of view.

ART (Advanced Radiation Therapy). The Gemelli ART is a highly specialized center where, besides providing both in and outpatients with the best clinical and latest technological treatment options, a dynamic research program is carried on inside our knowledge-based oncology laboratories. This research entity gathers several experts with different backgrounds (e.g., oncology, medical science, mathematics, physics, knowledge engineering) who are working together to enable the development of predictive models that will help physicians in the daily clinical practice.

Due to the fact that this is an observational research, patients do not have to be exposed to any additional exams or procedures beside the standard clinical practice. Minimal requirements for each participating center are the possibility to provide an electronic medical system to record patient's information, the ability to translate local data into the created ontology-based archive and to anonymize them. The availability of technology to conduct advanced multicenter research is also mandatory to be part of this project. Resourches to employ a data manager will be fundamental to continuously perform computerized and manual data consistency checks during data collection.

The primary objective of this research is to describe the development of a SDC model in order to obtain a national-based database. Originally set up inside the validation of high technology based on large database analysis by learning machine (VATE) project between the Policlinico Universitario Agostino Gemelli in Rome and the MAASTRO Clinic in Maastricht [40], this project is now continuing inside the European Registration Cancer Care (EURECCA) - Italia project and under the sponsorship of VARIAN Medical Systems. Conceived to reduce the differences between European countries, the international, multidisciplinary, outcome-based quality improvement European Registration Cancer Care program $[41,42]$, has been translated into the Italian setting with the primary goal to support practice survey and to develop and validate multifactorial prediction models for the different treatment outcomes will be considered (e.g., local recurrence, distant metastasis, overall survival, 
toxicities) by the analysis of an 'Italian large database'. Furthermore, the long-term aim is to build a DSS in order to personalize treatments both in terms of treatment's efficacy and treatment's toxicity control. Stratifying the different risk's classes related to the outcomes each times identified, DSS could be also useful to identify the best patients to be included in future randomized clinical studies. In addition, data on survival and toxicity can be used to compare the results of new and emerging radiation delivery techniques, targeted therapies or chemotherapy regimens after they have been clinically introduced to the results obtained with the standard treatment (Figure 3) [43].

The general hypothesis is that the performance improvement of the prediction models is related to the development of multifactorial models. The basic multifactorial models will be based on patient-related variables (e.g., age, sex), clinical presentations of the disease (e.g., staging, markers, imaging data), treatment data (e.g., chemotherapy, radiotherapy, surgery information, palliative care) and imaging data (diagnostic, treatment or follow-up images). The improved multifactorial models will include additional clinical and treatment imaging and/or genetic information as biological data is expected to be collected more and more in future routine practice.

\section{- SDC collection framework}

SDC's framework can be organized in several layers:

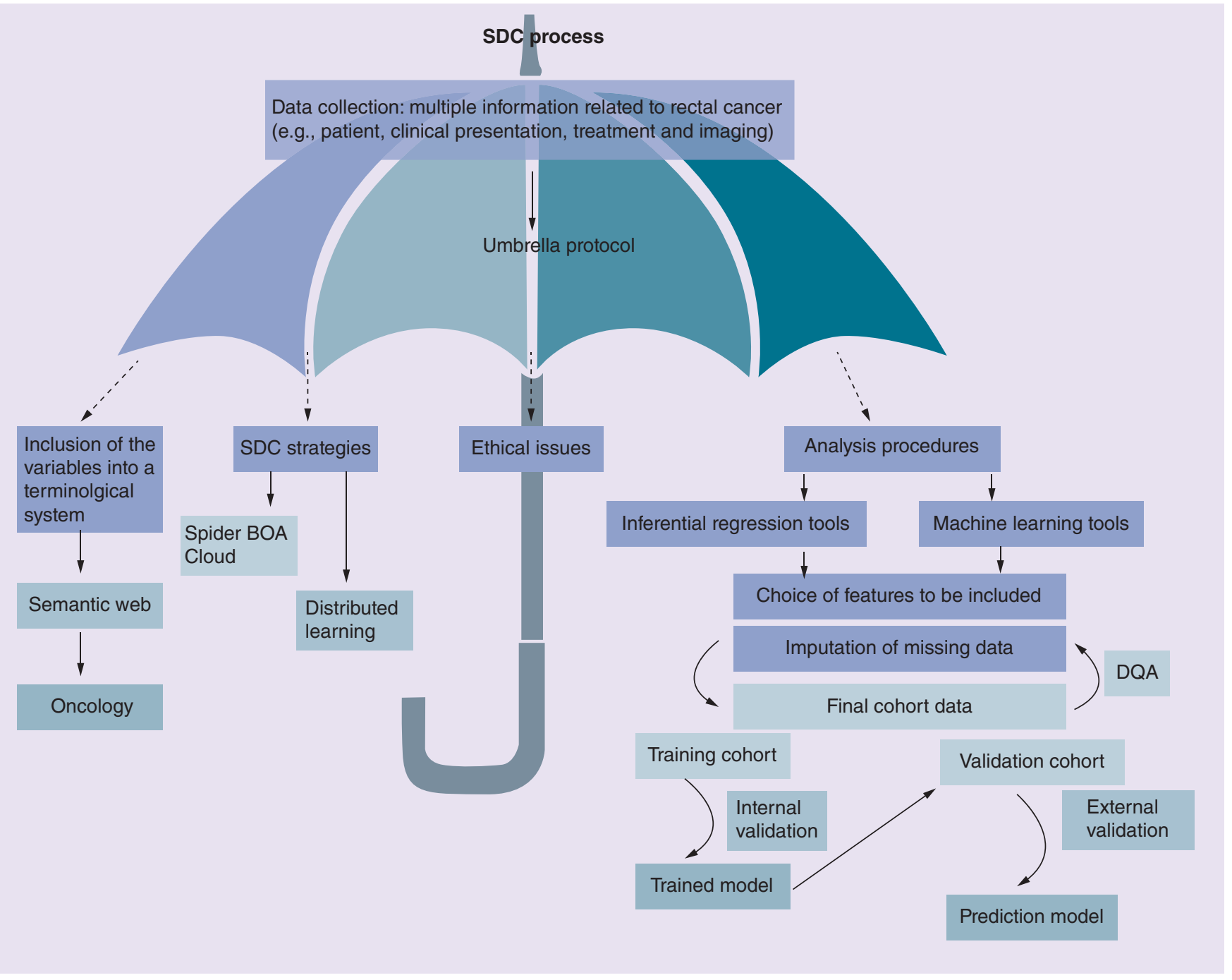

Figure 2. Standardized data collection process.

DQA: Data quality assurance; SDC: Standardized data collection. 


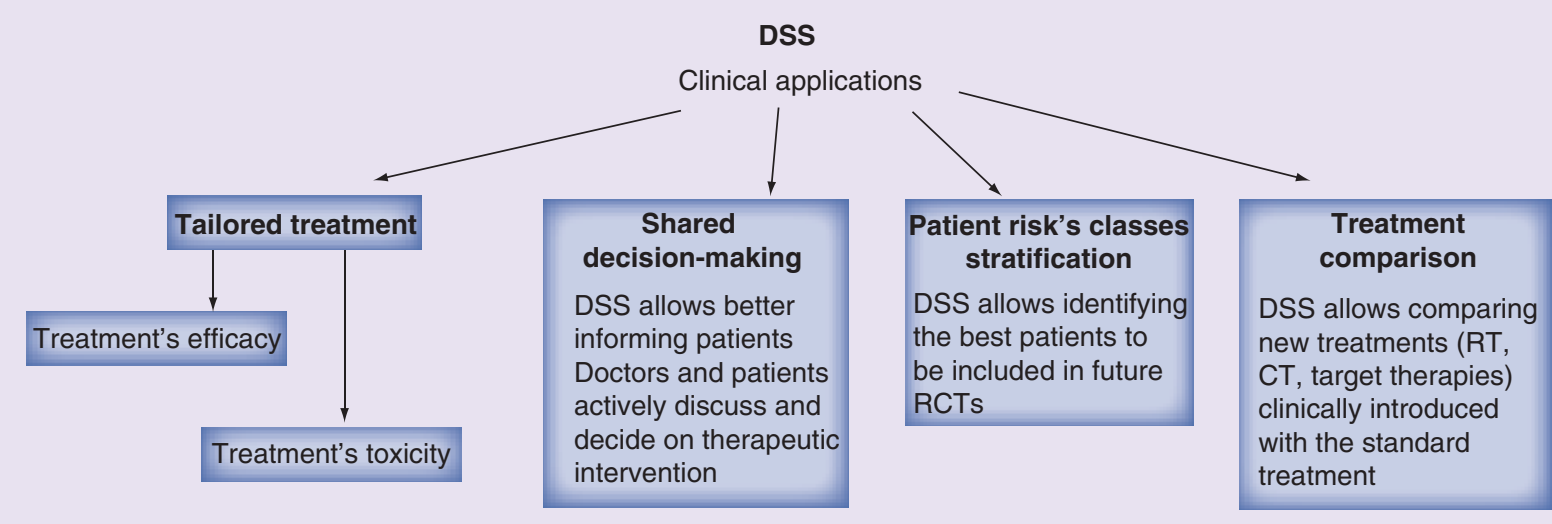

DSS

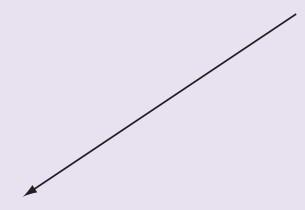

Clinical representation

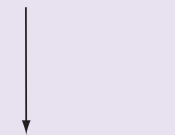

Graphical calculating devices

(e.g. nomograms)

Specific application for devices of

new generation (cell phone, tablet)

Interactive websites

(Table 1)

Figure 3. Decision support system: clinical application and representation.

CT: Chemotherapy; DSS: Decision support system; RCT: Randomized clinical trial; RT: Radiotherapy.

- Patient's privacy has to be protected;

- The development and validation of multifactorial prediction models require the availability of large amount of data considered significant for present and future studies;

- Each variable has to be included into a terminological system. Adding more variable in the future is possible, but starting early with most important variables is fundamental;

- Provenance (e.g. previously discussed temporal and geographical aspects) needs to be included in the data, to enable secondary use of routine clinical data;

- Reusability of legacy data is possible, at the condition that suitable semantic remapping functions from old to new data are provided;

- Appropriate mathematical and statistical methods are needed in order to learn from a large collection of data (large database) and aid in testing new modeling-based hypotheses.

\section{- SDC general}

All patients with a diagnosis of rectal cancer will be eligible for the inclusion in the SDC.
The standardization includes both a retrospective and a prospective assessment of baseline characteristics and treatment-related factors (including dose distribution parameters, acute and late radiation-induced toxicity, local control, disease-free survival, overall survival and health-related quality of life). Additional information on radiotherapy will be extracted in an automated way from the radiotherapy record and verification information system. More detailed information regarding dosimetric parameters can be calculated using the 3D dose matrix and the imaging information. Acute and late toxicity will be scored according to the Radiation Therapy Oncology Group scale (for the retrospective analysis) and to the CTCAE v3.0 or CTCAE v4.0 (for the prospective analysis). Finally, quality of life will be measured using the EuroQol-5D-5L, EORTC QLQ-C30 and EORTC QLQ-C29 questionnaires.

The use and role of medical imaging technologies in clinical oncology has passed from a primarily diagnostic, qualitative tool towards a central role in the context of individualized medicine with a quantitative value. Several studies, such as radiomics $[44,45]$, have been 
developed to analyze and quantify different imaging features (e.g., descriptors of intensity distribution, spatial relationships between the various intensity levels, texture heterogeneity patterns, descriptors of shape, among others) and their correlations with tumor and surrounding tissues. Furthermore, these studies attempted to identify relationships with treatment outcomes or gene expressions. To be useful in a rapid-learning health setting, diagnostic, treatment and follow-up imaging information must be automatically retrieved from the source to pre-compute these radiomics features, and to subsequently use these features in prognostic models.

\section{- SDC strategies to implement prediction models for rectal cancer}

The availability of multiple clinical data, together with improved imaging modalities, leads to large amounts of medical and biological data, which are most effectively analyzed using computational methods. Beside traditional statistical tools (e.g., [generalized] linear models and/or survival models), machine learning methods can be applied to facilitate development of more advanced and complex prediction models. Iteratively learning from data through specific algorithms, machine learning is able to train a computer how to unearth hidden connections without knowing exactly where to look. There are a number of different techniques [15] for this purpose, mainly Bayesian networks [46,47] or support vector machines [21]. These techniques can overcome problems encountered with conventional statistical methods especially if data are highly correlated, many variables are available but a limited number of patients (high-dimensional data), or many different models have to be tested for their predictive value. In the field of radiotherapy and especially for the prediction of treatment responses, such machine learning techniques are becoming more apparent. Successes over traditional statistics have already been published [48] and first promising results for building predictive models concerning survival of rectal cancer patients are already found in the literature $[21,49]$.

\section{- Main SDC strategies}

To accomplish the issue of collecting large amount of data, two different strategies will be used in our institution depending on the research's purpose and centers' agreement. On the one hand a centralized data records' consolidation approach will first convert data archives accordingly to a global data dictionary (e.g. an ontology) and then it will transfer the anonymous reproduction of the clinical data into a cloud-based large database. This is the line with the standard extract-transferload (ETL) tools originanting from the field of Buisness Intelligence (BI). An alternative called distributed learning is a very flexible approach that allows to learn from the data without the need for data to leave the individual hospital. Nonetheless, semantic interoperability (in terms of data structure and terminology) is still needed in the latter situation [49]. In the following paragraphs, these two approaches will be described in more detail.

\section{Centralized consolidation of data records} approach (Spider BOA Cloud)

A software called 'Spider BOA' (System for Patient Individual Data Entry and Recording Beyond Ontology Awareness), which is part of a commercial electronic medical record system has been developed for Radiation Oncology wards. The information technology architecture of Spider BOA converts (legacy) archives of a center using a global data dictionary and replicates anonymously the clinical data into a cloud-based large database (Figure 4). The Global Data Dictionary is designed to be compatible with the standard Clinical Data Interchange Standards Consortium Operational Data Model to exchange data in a common format.

The cloud-based large database is the only asset that is shared among participating centers; this sharing is only temporary, research-bound and lasts through the life span of a particular study. The system guarantees that nothing, except anonymous and nonreferable clinical data - with no link to the original local archives - are part of the large database.

Furthermore, to investigate a predictive model by using information from one or more institutes, statistical algorithms will be run through the aggregated data, in a process which needs not and does not give access to individual records or multiple records regarding the same physical individual.

The supervisor center can only query the shared large database, complying with the requests of researchers and giving back results 


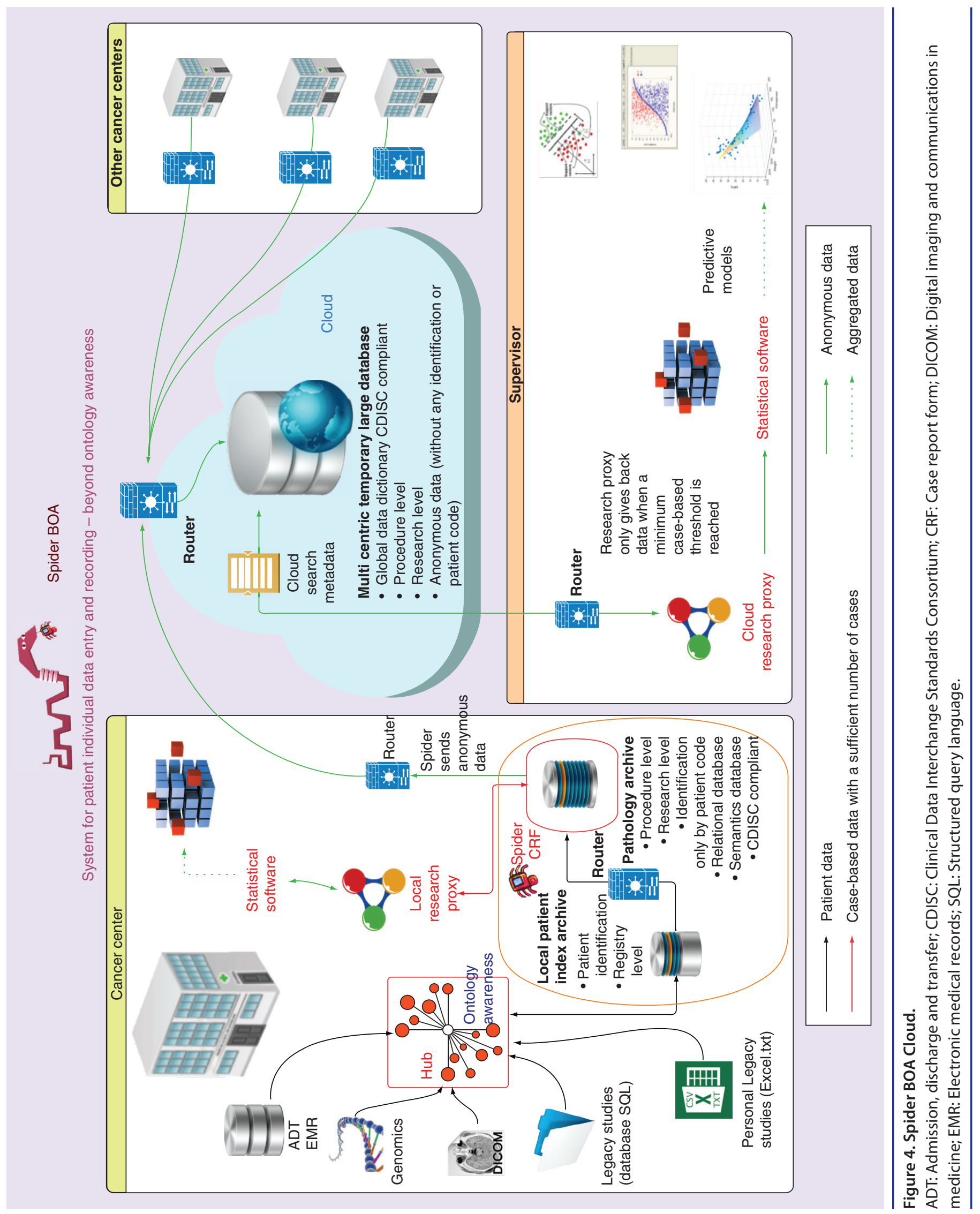


according to the policies of the participating centers. At the end of the study life span, the cloud database is deleted.

Each center can make local queries on its own database, similar to how the research supervisor runs queries on the centralized database and calculate results. A software middleware, called Research Proxy, is located inside the walls of each institution, and acts as an interface between the local repository and the research supervisor. Its main roles are to present the supervisor with a standardized representation of the data provided by the institution and to answer metadata queries (e.g., numbers of data records and their quality). It can also perform data projection, consolidation and filtering at a local level, while ensuring that privacy is enforced by filtering out all patient identifiable information before it leaves the institution. Data subsets produced by the Research Proxy can be used to build and validate prognostic models.

In a second stage, the system shall evolve toward a distributed learning approach: the supervisor's learning analyzer proxy will send algorithms directly to local research proxies, taking back from them only the results of each iteration step, with no need to work with shared data in the cloud anymore. We will elaborate more on this distributed learning approach in the next paragraph.

\section{Distributed learning approach}

During recent years, many issues regarding data property and privacy have challenged the feasibility of multicentric research projects based on large data collections. This aspect is of the utmost importance when patient data are involved.

A possible solution is found in the distributed learning paradigm, consisting of a set of technologies and infrastructures that allow statistical and/or machine learning without exchanging patient identifiable information among participating institutes.

In a distributed learning architecture, each research institution runs a local application on its data, and sends the resulting model to a master application (proxy in Figure 5), located on a central server. At this central server, all proposed models are compared in order to build a new iteration of the process, until convergence is reached around a shared model. Convergence is usually achieved after a few hundred iterations; the process is fully automated and all data exchange can be encrypted with ease, although no data stream contains information on an individual level.

This architecture has been successfully tested and found working well for a variety of learning algorithms [50].

In distributed learning mode, local research proxies do not move data around: they only apply iterative algorithms that the supervisor will use to build consensus and estimate the model's parameters. Presently, some of the most common predictive models used in statistics, the Cox proportional hazard models, cannot be run in a distributed learning framework. Hence, this means that prediction models presenting probabilities multiple future time points are not (yet) feasible in a distributed learning setting.

The technology to apply distributed learning requires the availability of an appropriate information technology platform to allow a seamless, real-time bidirectional data flow between each institution and the supervisor. Available bandwidth is not of great importance, as patient data are not traveling across the infrastructure and the packets transferred at each step in the iterative process are relatively small (kilobites). Still, for each participating center, an open connection to the research proxy, through the institution's firewall, is an expressed requirement. Furthermore, a reliable and stable connection is needed in order to execute all iterations.

A first implementation of the system has already been tested as a proof of concept and is currently in the prototyping phase inside the 'VAlidation of TEchnology in radiotherapy (VATE)' project [40,51].

\section{- Semantic Web technology}

The transition from a list of variables identified as relevant for rectal cancer to an ontology, requires the increase of both the complexity and the formalism of the language with the usage of more complex constructs to represent relationships between variables and enriching knowledge contents. Semantic Web technology [52] can be used to describe data according to a pre-defined ontology, and is flexible in terms of describing additional information. This technology is governedand maintained by the World Wide Web Consortium (W3C), which among others manages the standards for the 


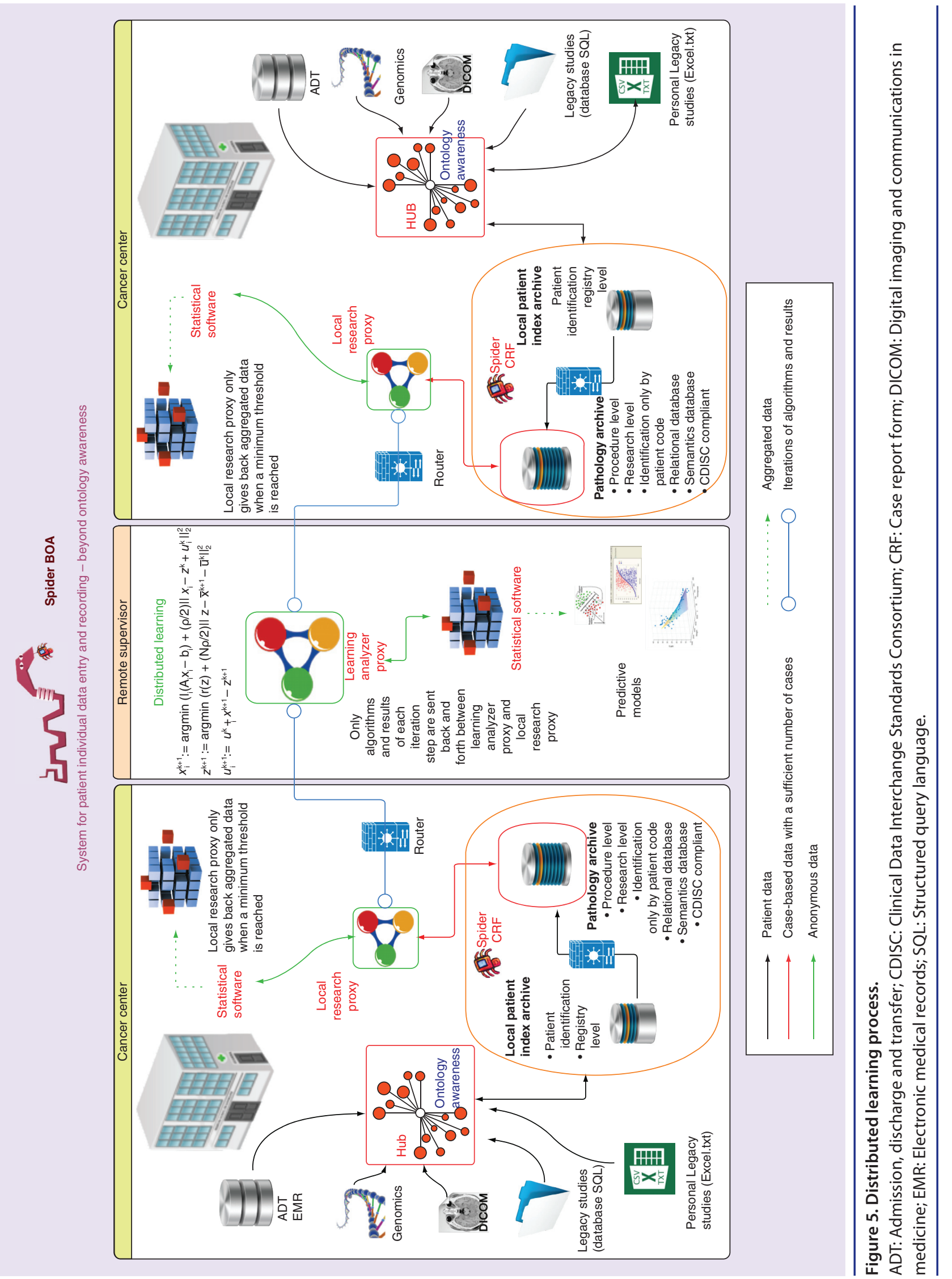


web (HTTP). For the Semantic Web technology, data are represented using the Resource Description Framework (RDF) language [53]. This framework basically consists of one table with three columns (column names: subject, predicate and object), where every row is a triple, defining a source (subject), target (object) and an arrow having semantics on its representation. Where relational databases are built around tables and references, RDF is a graph database to build a web (graph) of data. The interaction between elements of multiple triplets is defined inside an ontology. To infer information using a pre-specified description logic, more advanced languages have been built on top of RDF (RDFS or OWL). Software, built to interpret the ontology, can easily parse the (inferred) data and can be used on large (distributed) repositories to learn prediction models.

Beside the RDF representation of Semantic Web which is both extremely simple and flexible (one table with three columns), the federated nature of the web with both data and knowledge residing at multiple (geographically separated) internet locations and the possibility to query them all at once using SPARQL (the most common query language of the Semantic Web [54]), justifies the great power of the Semantic Web technology.

\section{- SDC statistical analysis}

Data analysis features

After data extraction and querying is implemented, prediction models can be built using two large families of data analysis tools: the classical statistical analysis and the machine learning tools. The former includes data modeling via generalized linear or survival models based typically on traditional approaches like maximum likelihood; the latter approach can delve deeper into hidden relationships among covariates and between covariates and outcomes, and are based on supervised (e.g., Bayesian networks [46,47], support vector machines [21]) and unsupervised (e.g., clustering [55]) algorithms.

All developed prognostic models, must undergo a strict evaluation process mainly based on internal and external validation [56] in order to become a reliable tool to be used in clinical contexts and reported according to TRIPOD recommendation [29].

The methodological process to learn, in other words, to go from data to useful decision support is described below.
First, data quality (e.g., completeness and accuracy) must be assessed, then relevant covariates are selected according to specific research aims and importance. Data quality can then be improved, for example, by imputation (for missing data), and bias (e.g. selection or observer bias) can be detected and optionally corrected.

Independently from the model of choice, available data are split into a training set and a validation set. The learning effort is conducted on the training set, and then internally validated on the validation set. Model performance can be assessed in terms of calibration (graphically determined, or via a Hosmer-Lemeshow test) and discrimination, by using the area under the receiver operating curve (AUC) or c-statistics.

Finally, model performance must be externally (indipendently) validated by applying the trained prediction model on the external dataset by measuring model performance as described above.

After validation of the model performance, decision curve analysis [57,58] can be used to assess the clinical value of the model and to determine probability thresholds when implementing in clinical practice or DSS.

The final model can be presented to the end user in a variety of ways such as nomograms [1] or via interactive websites; depending on how the model fits in the clinical process.

\section{- Missing data}

The system will take advantage of a missing data tool (e.g., as a service, stand-alone application or embedded inside a learning algorithm) with the basic requirement of detect, report and impute missing data. For the detection a solution based on shape expressions [59] will be further developed. In this approach a data shape can be intuitively defined including taking full advantage of ontologies. This data shape can be converted into a SPARQL query that can query a local RDF store for all patients that fit the data shape. It can also query for the inverse (i.e., all patients that do not fit a data shape) and can guide which data elements (e.g., T-stage is missing) are not matching the required shape expression. The results of these data queries are the basis of the detection and reporting of missing data. The imputation of missing data is a research field by itself. A literature review will be performed to implement all 
common imputation methods (e.g., the investigators have used median, mean and Bayesian imputation in prior work) available and configurable in the context of a specific research question. The imputed data elements will be stored in a separate graph in the local RDF store including their provenance so that the imputed elements can be separated from the asserted data elements. Interfacing between the tool and the calling learning application will be defined during the project based on user requirements.

\section{- Control of data consistency}

The data manager will perform computerized and manual consistency checks by ad hoc retrieval services. A 'continuous data quality assurance' (CDQA) is of paramount importance since any prediction model is only as good as the data it was built upon.

CDQA is enforced at different levels: when an ontology is implemented, accepted values are declared for each covariate, both categorical and ranges, on the basis of accepted practice and plausibility criteria (e.g., T-stage can only be one of T1, T2, T3, T4; age will only be accepted in the range $0-140)$.

At the interface level, checks are conducted in real time to flag inappropriate or suspect inputs and allow for immediate correction.

At a higher level, frequentist or Bayesian approaches can be chosen to implement CDQA bots that scan the data in search of unlikely combinations to be scheduled for further investigation by domain experts. This is especially important in view of an ever growing, dynamic database, continuously populated by different sources located in different countries.

Such an approach can also spot legitimate but nevertheless adverse changes in data representation semantics that happen, for instance, when a staging system is officially changed, thus producing heterogeneous data collections, affecting overall information value detrimentally.

A final data quality check can be introduced at the modeling stage, in order to assure specific hypotheses either model-, pathology- or research (end point)-related.

Queries will be issued in case of inconsistencies. Consistent forms will be validated by the data manager. Inconsistent data will be kept 'on hold' until resolution of the inconsistencies.

\section{Ethical considerations}

\section{- Privacy protection of patients}

Patient accrual will be conducted by each center selecting those who meet the inclusion criteria. Written informed consent for anonymized treatment data collection and approval of related research for prospective patient accrual will be collected according to the different regulations of the participating centers.

In case of a distributed learning approach, patient's related data do not need to leave the institute. This is feasible as the central 'master' sends possible prediction models rather than fetching the data from remote nodes. Only statistical indexes unrelated to specific patients are exchanged between nodes and their master [50,60-61].

In a centralized data records' consolidation approach, patient's privacy will be protected at the architectural level because all data transfer will happen through a fully encrypted pipeline, and directly identifiable patient information (e.g., name, patient ID, address) will be anonymized before leaving the local center's walls. The mapping between local hospital ID and research ID is protected via software procedures and will never leave the originating center. Attempts of intercepting data is therefore challenging and will result in information unrelated to actual patients. In order to comply with local regulations or specific policies at the centers' level, a second level of encryption can be added by encrypting the data itself, next to the already encrypted pipeline.

\section{Data privacy strategy}

The risk of privacy infringement for participating institutes is minimized by the project. All data, originating from local repositories at the institute's site, will be routed to a local repository in totally anonymous form, because all names and links to traceable information will be removed before entering the repository, while unique identifiers that could point directly or indirectly to individual patients will be remapped to a different code.

As a consequence, the local end point, which is queried by other research group members from outside the institute during normal activity, will not expose any method to reconcile clinical information to the relevant patient. Under this point of view, our data will have a higher degree of protection than the same data stored in the institute's databases. 
It also must be noted that in investigating a predictive model by using information from one or more institutes, statistical algorithms will be run through the aggregated data, in a process which needs not and does not access individual records or multiple records regarding the same physical individual. The inherent statistical nature of this activity adds yet another level of protection to the system.

When end points are added locally, in order to enable data mining activities in a Semantic Web approach, all data will be anonymized and, when information is collected horizontally across different institutes, further levels of isolation will be achieved through data sectioning (e.g., stripping away the geographical localization of the patient and contributing institute) as seen necessary.

\section{Patient privacy data mining}

In both 'cloud' and 'distributed' ways, patient privacy is enforced at the architectural level because in a distributed learning approach, data never leave the hospital walls and in a centralized consolidation of data records, any reference to personal information is physically detached from each individual data record before it leaves the hospital walls [62].

Each record is assigned a new, randomly generated ID whose only purpose is to join records belonging to the same patient once the data record are collected at the master's level. The remapping function is deleted before the dataset leaves the originating hospital, hence the relation is broken and no backward mapping (i.e., from data to physical patient) is possible.

\section{Conclusion}

In oncology, the awareness of the complex responsibilities of the multiple factors involved in the clinical outcome and/or in the treatment's toxicity processes, makes clear how the human cognitive capacity is limited with the few variables discriminated and used in daily clinical practice [13,63-64].

The tremendous advances in diagnostic and treatment technologies and the great emphasis in the development of new therapeutic modalities with better defined/targeted mechanisms of action, have been the main characters of the fight against cancer, in the last 10 years. However, choosing the best therapeutic treatment from a range of options and patient characteristics is challenging.
Progress in computer technology with new diagnostic methods and treatment modalities development has been responsible for advances in radiation oncology with radiotherapy planning and delivery facilities. Although the progress in computer technology has had an important influence in radiotherapy planning and delivery facilities evolution and has allowed remarkable precision in treatment delivery and better outcome, the dose escalation process can increase severity and duration of side effects [65] and it is unknown in which patients such escalation might be a good option.

The use and role of medical imaging technologies in clinical oncology has also greatly expanded during the last decade from a primarily diagnostic, qualitative tool toward a central role in the context of individualized medicine with a quantitative value. Several studies have been developed to analyze and quantify different imaging features (e.g., descriptors of intensity distribution, spatial relationships between the various intensity levels, texture heterogeneity patterns, descriptors of shape, among others) and the relations of the tumor with the surrounding tissues to identify possible relationships with treatment outcomes or gene expressions [44,45].

However, prediction of outcome in order to choose the optimal treatment is complicated in view of the complex, dynamic nature of cancer and organs at risk. Therefore it becomes more and more apparent that certain subgroups of patients may benefit from a specific treatment, while others do not or even may have a worse outcome [66]. The same is observed for the toxicity of the treatment. Some patients suffer from severe side effects while others are relatively unaffected [67]. This means that there is a complex interplay of different factors which has not yet been unraveled. These differences between individual patients are not only observed in case of treatment with medication or chemotherapy, but they also occur both during radiotherapy treatment - implying that the decision to escalate the radiation dose should be individualized - and surgery (e.g., watchful waiting or surgical intervention).

DSS will enable to predict the outcome for a certain patient in combination with a specific treatment with more accuracy. It will lead to better identification of risk groups, which results in stage migration and consequently in the research of new therapies or different treatment options for these patients. Therefore, treatment 
will be more individualized as patients will be protected from excessive toxicity and discomfort and empowers the choice of the best treatment at hand for each patient. Currently, this choice is based on general guidelines that only take into account a low number of variables. These guidelines are developed for groups of patients and therefore can lead to overtreatment in some patients or inadequate therapy in others, resulting in major expense for individuals and society.

Studies, investigating the performance of physicians in predicting side effects of radiotherapy treatment show that human lack predictive ability [68]. Therefore, treatment can only become more personalized if accurate, scientifically based decision aids are developed, that can offer assistance in clinical decision-making in daily practice.

Finally, considering the important role that predictive models could play in the clinical practice, clinicians must be aware that they remain only decision support systems, not decision makers. Although they can be very useful with great performances and sometimes with a great $\mathrm{p}$-value, these models are characterized by intrinsic limits (e.g. the hard variables versus overall well-being of patient) and confidence intervals. An accurate internal and external validation is essential to evaluate the quality of the collected data and to support the general applicability of the same prediction models.

\section{Future perspective}

The amount of available information to explain the very complex, dynamic behavior of cancer is continuously expanding due to the tremendous improvement in diagnostic procedures such as genomic and proteomic profiling (e.g., based on the patient's blood or saliva), anatomical and functional imaging techniques (e.g., CT, MRI, PET) and treatment modalities.

Long considered to have only a physical role, radiation therapy is now conceived to act more as a biological intervention. Affecting tissues at cellular and molecular level, radiation activity should be modulated by biological signaling pathways and the immunological system [69,70]. Accordingly, a growing efficacy of the combination of radiation therapy with targeted biological agents has been already proved, increasing the awareness that holds promise for future advances [71,72]. New, less toxic anticancer therapies are being developed. They include new approaches targeting cancer-specific pathways in the cell and intending to improve the treatment outcome in terms of survival as well as toxicity [73,74].

Dose distribution is one of the main topics of radiation therapy's publications. It has been demonstrated that dose distribution can be easily fitted to complex anatomical shapes producing a dose distribution optimization but actually no one has shown consistent outcomes in terms of tumor control or organ at risk due to the small series of patients and the lack in homogeneity of data collection used in such clinical trials. Hence, it is necessary to create large databases, realized by crossing and combining multiple data sources already set in specific storage archives, to provide sufficient statistical power to act as acceptable decision supporting tools.

So far, the future of the cancer research is entrusted to the multidisciplinary groups able to deal with this hybrid discipline that encompasses all the different fields of oncology, computer science, bioinformatics, statistics, computational biology, genomics, proteomics, pharmacology, etc. The common goal is to bring order to the massive amount of data generated by the tremendous number of studies attempting to find the underlying causes and effective means of diagnosis and treating cancer [75]. Therefore, only by collaboration between these specialty disciplines, it will be possible to develop better preclinical models that will help physicians to choose the best treatment or the best combination of treatments for each patient in the most efficient possible way; by incorporating both clinical guidelines and predictive models.

Financial \& competing interests disclosure

The authors have no relevant affiliations or financial involvement with any organization or entity with a financial interest in or financial conflict with the subject matter or materials discussed in the manuscript. This includes employment, consultancies, honoraria, stock ownership or options, expert testimony, grants or patents received or pending, or royalties.

No writing assistance was utilized in the production of this manuscript.

\section{Open access}

This work is licensed under the Creative Commons Attribution-NonCommercial 4.0 Unported License. To view a copy of this license, visit http://creativecommons.org/ licenses/by-nc-nd/4.0/ 


\section{EXECUTIVE SUMMARY}

\section{Personalized therapy for rectal cancer}

- In the era of personalized

- medicine, the development of validated decision support systems (DSSs) beside the existing clinical guidelines can help physicians to personalize treatments both in terms of treatment's efficacy and treatment's toxicity control.

- Stratifying the different risk's classes, DSS could also be useful to identify the best patients to be included in future randomized clinical studies.

- Data on survival and toxicity can be used to compare the results of new and emerging radiation delivery techniques, targeted therapies or chemotherapy regimens with the standard treatment.

\section{Multifactorial predictive models: a continuing challenge}

- The wide tumor's heterogeneity and the well-known complexity of the clinical outcome and/or the treatment's toxicity, justify the progressive efforts in developing predictive models.

- The number of evaluated features coming from the integration of multiple disciplines of oncology, computer science, bioinformatics, statistics, genomics, imaging, among others, could be very large as to make the traditional statistical analysis difficult to achieve.

- Automated data-mining and processes and machine learning approaches can be a solution for bringing order to the massive amount of data, trying to unravel important interaction among model variables.

- Notwithstanding the development of high selective treatment, clinical data are still, in some cases, disappointing, justifying the development of predictive models.

\section{Ontology \& data standardization}

- The development of DSS implies a new concept of research where all the information have to be collected without knowing beforehand what the relevant features will be.

- Any considerable variable has to be standardized through a standardized data collection (SDC) process, in order to universally define data and procedures allowing automatic and consistent upload from any exploitable data source.

- SDC will benefit from using an ontology, a classification system where each variable can be represented using uniform and unambiguous definitions.

- Any ontology can grow from a simple dictionary to a more formal expression resulting from the sharing of the definition between different institutions.

\section{Umbrella protocol}

- The strategy to collect data in a standard and consistent manner and to analyze them properly for decision support is called 'umbrella protocol.'

- Umbrella protocol is represented by the set of materials and procedures related to a group of specific research projects.

- The main SDC strategies, the analysis procedures and the possible occurring ethical issues related to a specific research project, are described in an umbrella protocol.

\section{A rectal cancer's SDC prototype}

- An umbrella protocol related to the rectal cancer's SDC has been recently drafted in the radiotherapy department of the Policlinico A Gemelli in Rome with the primary objective to obtain a national-based database inside the 'European Registration Cancer Care - Italia' project.

- All patients with a diagnosis of rectal cancer will be included in SDC. The standardization includes both a retrospective and a prospective assessment of baseline characteristics and treatment-related factors.

- To accomplish the issue of collecting a large amount of data, two different strategies will be used depending on research's purpose and center's agreement:

- Centralized data records' consolidation approach (Spider BOA Cloud) - converts data archives accordingly to a global data dictionary and then it will anonymize them and transfer them into a cloud-based large database; 


\section{EXECUTIVE SUMMARY (CONT.)}

\section{A rectal cancer's SDC prototype (cont.)}

- Distributed learning approach - allows to learn from the data without the need for data to leave the individual hospital.

- The transition from a list of variables identified as relevant for rectal cancer to an ontology, requires the increase of both the complexity and the formalism of the language. Semantic Web technology is the model used to represent data distribution.

- Its power is the simple however flexible representation and the federated nature of the web where data can be queried using Simple Protocol and Resource Description Framework Query Language.

- The two large families of data analysis tool used to build prediction models: the classical statistical analysis and the machines learning tool.

- Each model must undergo an internal and external validation in order to become a reliable tool to be used in clinical context and reported according to the Transparent Reporting of a multivariable prediction model for Individual Prognosis Or Diagnosis recommendation.

- The system will take advantage of a missing data tool to detect, report and impute missing data.

- The data manager will perform computerized and manual consistency checks by a 'continuous data quality assurance' process.

- In both 'cloud' and 'distributed' ways, patient's confidential information will be enforced at the architectural level without any increased risk of privacy infringement.

\section{Future perspective}

- The future of the cancer research is entrusted to the multidisciplinary groups with the common goal to develop multifactorial predictive models ables to unravel important nonlinear interaction among model variables.

\section{References}

Papers of special note have been highlighted as:

- of interest; $\bullet$ of considerable interest

1 Valentini V, van Stiphout RGPM, Lammering G et al. Nomograms for predicting local recurrence, distant metastases, and overall survival for patients with locally advanced rectal cancer on the basis of European randomized clinical trials. J. Clin. Oncol. 29(23), 3163-3172 (2011).

2 Cheung WY, Shi Q, O'Connell M et al. The predictive and prognostic value of sex in early-stage colon cancer: a pooled analysis of 33,345 patients from the ACCENT database. Clin. Colorectal Cancer 12(3), 179-187 (2013).

3 Bowles TL, Hu C-Y, You NY, Skibber JM, Rodriguez-Bigas MA, Chang GJ. An individualized conditional survival calculator for patients with rectal cancer. Dis. Colon Rectum 56(5), 551-559 (2013).

4 Peng J, Ding Y, Tu S et al. Prognostic nomograms for predicting survival and distant metastases in locally advanced rectal cancers. PLoS ONE 9(8), e106344 (2014).

5 Van Stiphout RGPM, Valentini V, Buijsen J et al. Nomogram predicting response after chemoradiotherapy in rectal cancer using sequential PETCT imaging: a multicentric prospective study with external validation. Radiother. Oncol. 113(2), 215-222 (2014).

6 Battersby N, Bouliotis G, Emmertsen K, Laurberg S, Moran B. OC-097 An externally validated nomogram to predict the risk of bowel dysfunction following an anterior resection. Gut 64(Suppl. 1), A48.2-A48 (2015).

7 Weiser MR, Landmann RG, Kattan MW et al. Individualized prediction of colon cancer recurrence using a nomogram. J. Clin. Oncol. 26(3), 380-385 (2008).

8 Valentini V, Lambin P, Myerson RJ. Is it time for tailored treatment of rectal cancer? From prescribing by consensus to prescribing by numbers. Radiother. Oncol. 102(1), 1-3 (2012).

9 Sullivan R, Peppercorn J, Sikora K et al. Delivering affordable cancer care in high-income countries. Lancet. Oncol. 12(10), 933-980 (2011).

10 Booth CM, Tannock IF. Randomised controlled trials and population-based observational research: partners in the evolution of medical evidence. Br. J. Cancer $110(3), 551-555$ (2014).

11 Lambin P, Zindler J, Vanneste B et al. Modern clinical research: how rapid learning health care and cohort multiple randomised clinical trials complement traditional evidence based medicine. Acta Oncol. 54(9), 1289-1300 (2015).

12 Lambin P, Roelofs E, Reymen B et al. 'Rapid learning health care in oncology' - an approach towards decision support systems enabling customised radiotherapy. Radiother. Oncol. 109(1), 159-164 (2013).

13 Abernethy AP, Etheredge LM, Ganz P a et al. Rapid-learning system for cancer care. J. Clin. Oncol. 28(27), 4268-4274 (2010).

- Analysis of the elements of a rapid-learning system for cancer to better understand the state of the rapid-learning healthcare model and its potential implication on oncology.

14 Naqa IEl, Deasy JO, Mu Y et al. Datamining approaches for modeling tumor control probability. Acta Oncol. 49(8), 1363-1373 (2010)

-. The prediction of treatment response can be improved by utilizing data mining approaches, which have the capacity to predict on unseen data for prospective clinical applications.

15 Kang J, Schwartz R, Flickinger J, Beriwal S. Machine learning approaches for predicting 
radiation therapy outcomes: a clinician's perspective. Int. J. Radiat. Oncol. Biol. Phys. 93(5), 1127-1135 (2015).

16 Burman C, Kutcher GJ, Emami B, Goitein M. Fitting of normal tissue tolerance data to an analytic function. Int. J. Radiat. Oncol. Biol. Phys. 21(1), 123-135 (1991).

17 Gay HA, Niemierko A. A free program for calculating EUD-based NTCP and TCP in external beam radiotherapy. Phys. Med. 23(3-4), 115-125 (2007).

18 Gorlia T, Stupp R, Brandes A et al. New prognostic factors and calculators for outcome prediction in patients with recurrent glioblastoma: a pooled analysis of EORTC Brain Tumour Group Phase I and II clinical trials. Eur. J. Cancer 48(8), 1176-1184 (2012).

19 Weiser MR, Gönen M, Chou JF, Kattan MW Schrag D Predicting survival after curative colectomy for cancer: individualizing colon cancer staging. J. Clin. Oncol. 29(36), 4796-4802 (2011).

20 Chen S, Wang WBroman KWKatki HA Parmigiani G Bayes Mendel: an R environment for Mendelian risk prediction. Stat. Appl. Genet. Mol. Biol. 3, Article 21 (2004).

21 Van Stiphout RGPM, Lammering G, Buijsen J et al. Development and external validation of a predictive model for pathological complete response of rectal cancer patients including sequential PET-CT imaging. Radiother. Oncol. 98(1), 126-133 (2011).

22 Prognostic Calculators. www.eortc.org

23 Prediction Tools - A Tool for Doctors and Patients.

www.mskcc.org/nomograms

24 BayesMendel Lab. http://bcb.dfci.harvard.edu

25 LifeMath. www.lifemath.net

26 Cancer Risk Assessment tool (RAT). www.cancerresearchuk.org

27 Cancer Risk Prediction and Assessment. http://epi.grants.cancer.gov

28 MAASTRO. www.predictcancer.org

29 Collins GS, Reitsma JB, Altman DG, Moons KGM. Transparent Reporting of a multivariable prediction model for Individual Prognosis Or Diagnosis (TRIPOD): the TRIPOD Statement. Br. J. Surg. 102(3), 148-158 (2015).

- The transparent reporting of a multivariable prediction model for Individual Prognosis
Or Diagnosis statement aims to improve the transparency of the reporting of a prediction model study regardless of the study methods used.

30 Gorlia T, van den Bent MJ, Hegi ME et al. Nomograms for predicting survival of patients with newly diagnosed glioblastoma: prognostic factor analysis of EORTC and NCIC trial 26981-22981/CE.3. Lancet Oncol. 9(1), 29-38 (2008).

31 Jaspers MWM. A comparison of usability methods for testing interactive health technologies: methodological aspects and empirical evidence. Int. J. Med. Inform. 78(5), 340-353 (2009).

32 Valentini V, Aristei C, Glimelius B et al. Multidisciplinary Rectal Cancer Management: 2nd European Rectal Cancer Consensus Conference (EURECA-CC2). Radiother. Oncol. 92(2), 148-163 (2009).

33 Haustermans K, Debucquoy A, Lambrecht M. The ESTRO Breur Lecture 2010: toward a tailored patient approach in rectal cancer. Radiother. Oncol. 100(1), 15-21 (2011).

34 Lambin P, van Stiphout RG, Starmans MH et al. Predicting outcomes in radiation oncology--multifactorial decision support systems. Nat. Rev. Clin. Oncol. 10(1), 27-40 (2013)

-• Overview of the factors that are correlated with outcome - including survival, recurrence patterns and toxicity - in radiation oncology and discuss the methodology behind the development of prediction models.

35 Keizer NF De. Understanding Terminological Systems I: terminology and typology. Methods Inf. Med. 39, 16-21 (2000).

36 Keizer NF De. Understanding Terminological Systems II: experience with conceptual and formal representation of structure. Methods Inf. Med. 39, 22-29 (2000).

37 CancerData.org. Protocols. www.cancerdata.org/protocols

38 Meldolesi E, van Soest J, Dinapoli N et al. Medicine is a science of uncertainty and an art of probability (Sir W. Osler). Radiother. Oncol. 114(1), 132-134 (2015).

39 Meldolesi E, van Soest J, Dinapoli N et al. An umbrella protocol for standardized data collection (SDC) in rectal cancer: a prospective uniform naming and procedure convention to support personalized medicine. Radiother. Oncol. 112(1), 59-62 (2014).

- Description of the main features of an umbrella protocol for standardizing data and procedures to create a consistent dataset useful to obtain a trustful analysis for a decision support system for rectal cancer.

40 Meldolesi E, van Soest J, Alitto AR et al. VATE: VAlidation of high TEchnology based on large database analysis by learning machine. Colorectal Cancer 3(5), 435-450 (2014).

41 Van de Velde CJH, Boelens PG, Borras JM et al. EURECCA colorectal: multidisciplinary management: European consensus conference colon \& rectum. Eur. J. Cancer 50(1), 1.e1-1.e34 (2014).

42 Breugom AJ, Boelens PG, van den Broek CBM et al. Quality assurance in the treatment of colorectal cancer: the EURECCA initiative. Ann. Oncol. 25(8), 1485-1492 (2014).

43 Harris AD, McGregor JC, Perencevich EN et al. The use and interpretation of quasiexperimental studies in medical informatics. J. Am. Med. Inform. Assoc. 13(1), 16-23 (2006).

44 Kumar V, Gu Y, Basu S et al. Radiomics: the process and the challenges. Magn. Reson. Imaging 30 (9), 1234-1248 (2012).

45 Lambin P, Rios-Velazquez E, Leijenaar $\mathrm{R}$ et al. Radiomics: extracting more information from medical images using advanced feature analysis. Eur. J. Cancer 48(4), 441-446 (2012).

46 Jayasurya K, Fung G, Yu S et al. Comparison of Bayesian network and support vector machine models for two-year survival prediction in lung cancer patients treated with radiotherapy. Med. Phys. 37(4), 1401-1407 (2010).

47 Oh JH, Craft J, Al Lozi R et al. A Bayesian network approach for modeling local failure in lung cancer. Phys. Med. Biol. 56(6), 1635-1651 (2011).

48 Cruz JA, Wishart DS. Applications of machine learning in cancer prediction and prognosis. Cancer Inform. 2, 59-77 (2006).

49 van Soest JPA. Dekker ALAJ Roelofs EGN. Machine Learning in Radiation Oncology. El Naqa I, Li R, Murphy MJ (Eds). Springer International Publishing, Switzerland (2015).

50 Boyd S. Distributed optimization and statistical learning via the alternating direction method of multipliers. Found. Trends Mach. Learn. 3(1), 1-122 (2010).

51 Valentini V, Dinapoli N, Damiani A. The future of predictive models in radiation oncology: from extensive data mining to reliable modeling of the results. Future Oncol. 9(3), 311-313 (2013).

52 Berners-Lee Tim, Hendler James LO. "The Semantic Web:" a new form of web content that is meaningful to computers will unleash 
a revolution of new possibilities. Sci. Am. Mag. (2001).

www.scientificamerican.com

53 Graham Klyne JJC. Resource Description Framework (RDF): concepts and abstract syntax (2004).

www.w3.org

54 Assélé Kama A, Choquet R, Mels G, Daniel C, Charlet J, Jaulent M-C. An ontological approach for the exploitation of clinical data. Stud. Health Technol. Inform. 192, 142-146 (2013).

55 Kaufman DA. Finding Groups in Data. John Wiley \& Sons, Inc., Hoboken, NJ, USA (2005).

56 Harrel FE Jr. Resampling, validating, describing, and simplifying the model. In: Regression Modeling Strategies: With Applications to Linear Models, Logistic and Ordinal Regression, and Survival Analysis. Springer International Publishing, Switzerland, 87-103 (2001).

57 Vickers AJ, Cronin AM, Elkin EB, Gonen M. Extensions to decision curve analysis, a novel method for evaluating diagnostic tests, prediction models and molecular markers. BMC Med. Inform. Decis. Mak. 8, 53 (2008).

58 Vickers AJ, Elkin EB. Decision curve analysis: a novel method for evaluating prediction models. Med. Decis. Making 26(6), 565-574 (2006).

59 Boneva I, Gayo JEL, Hym S, Prud'hommeau EG, Solbrig H, Staworko S. Validating RDF with shape expressions. arXiv 1404, 1270 (2014).

60 Wu Y, Jiang X, Kim J, Ohno-Machado L. Grid Binary LOgistic REgression (GLORE): building shared models without sharing data. J. Am. Med. Inform. Assoc. 19(5), 758-764 (2012).
61 Liu K, Kargupta H, Member S, Ryan J. Random projection-based multiplicative data perturbation for privacy preserving distributed data mining. IEEE Trans. Knowl. Data Eng. 18(1), 92-106 (2006).

62 Damiani A, Vallati M, Gatta R et al. Distributed learning to protect privacy in multi-centric clinical studies. Springer International Publishing, Switzerland (2015)

63 Chow E, Harth T, Hruby G, Finkelstein J, Wu J, Danjoux C. How accurate are physicians' clinical predictions of survival and the available prognostic tools in estimating survival times in terminally ill cancer patients? A systematic review. Clin. Oncol. (R. Coll. Radiol.) 13(3), 209-218 (2001).

64 Chow E, Davis L, Panzarella T et al. Accuracy of survival prediction by palliative radiation oncologists. Int. J. Radiat. Oncol. Biol. Phys. 61(3), 870-873 (2005).

65 Bentzen SM, Trotti A. Evaluation of early and late toxicities in chemoradiation trials. J. Clin. Oncol. 25(26), 4096-4103 (2007).

66 Mok TS, Wu Y, Thongprasert S et al. Gefitinib or carboplatin-paclitaxel in pulmonary adenocarcinoma. N. Engl. J. Med. 361(10), 947-957 (2009).

67 Bentzen SM, Hendry HJ. Variability in the radiosensitivity of normal cells and tissues. Report from a workshop organised by the European Society for Therapeutic Radiology and Oncology in Edinburgh, UK, 19 September 1998. Int. J. Radiat. Biol. 75(4), 513-517 (1999).

68 Oberije C, Nalbantov G, Dekker A et al. A prospective study comparing the predictions of doctors versus models for treatment outcome of lung cancer patients: a step toward individualized care and shared decision making. Radiother. Oncol. 112(1), 37-43 (2014).

69 Bentzen SM. Preventing or reducing late side effects of radiation therapy: radiobiology meets molecular pathology. Nat. Rev. Cancer 6(9), 702-713 (2006).

70 Fowler JF. 21 years of biologically effective dose. Br. J. Radiol. 83(991), 554-568 (2010).

71 Glynne-Jones R, Hadaki M, Harrison M. The status of targeted agents in the setting of neoadjuvant radiation therapy in locally advanced rectal cancers. J. Gastrointest. Oncol. 4(3), 264-284 (2013).

72 Bonner JA, Harari PM, Giralt J et al. Radiotherapy plus cetuximab for locoregionally advanced head and neck cancer: 5-year survival data from a phase 3 randomised trial, and relation between cetuximab-induced rash and survival. Lancet Oncol. 11(1), 21-28 (2010).

73 Bentzen SM, Harari PM, Bernier J. Exploitable mechanisms for combining drugs with radiation: concepts, achievements and future directions. Nat. Clin. Pract. Oncol. 4(3), 172-180 (2007).

74 Provencio M, Sánchez A, Garrido P, Valcárcel F. New molecular targeted therapies integrated with radiation therapy in lung cancer. Clin. Lung Cancer 11(2), 91-97 (2010).

75 Jiang H, An L, Baladandayuthapani V, Auer PL. Classification, predictive modelling, and statistical analysis of cancer data (a). Cancer Inform. 13(Suppl. 2), 1-3 (2014)

- Presentation of cancer informatics, a hybrid discipline encompassing several disciplines with the description of their role in finding answer in the fight against cancer. 